\title{
Deterministic scRNA-seq of individual intestinal organoids reveals new subtypes and coexisting distinct stem cell pools
}

\author{
Johannes Bues ${ }^{1,2,8}$, Marjan Biočanin ${ }^{1,2,8}$, Joern Pezoldt ${ }^{1,2}$, Riccardo Dainese ${ }^{1,2}$, Antonius \\ Chrisnandy ${ }^{3}$, Saba Rezakhani ${ }^{3}$, Wouter Saelens ${ }^{4,5}$, Vincent Gardeux ${ }^{1,2}$, Revant Gupta ${ }^{2,6}$, Julie \\ Russeil $^{1,2}$, Yvan Saeys ${ }^{4,5}$, Esther Amstad ${ }^{7}$, Manfred Claassen ${ }^{2,6}$, Matthias Lutolf $^{3}$, Bart \\ Deplancke $e^{1,2, *}$
}

1 Laboratory of Systems Biology and Genetics, Institute of Bioengineering, School of Life Sciences, Ecole Polytechnique Fédérale de Lausanne (EPFL), Lausanne, Switzerland

2 Swiss Institute of Bioinformatics (SIB), Lausanne, Switzerland

3 Laboratory for Stem Cell Bioengineering, Institute of Bioengineering, School of Life Sciences, Ecole Polytechnique Fédérale de Lausanne (EPFL), Lausanne, Switzerland

4 Data mining and Modelling for Biomedicine, VIB Center for Inflammation Research, Ghent, Belgium

5 Department of Applied Mathematics, Computer Science and Statistics, Ghent University, Ghent, Belgium

6 Institute for Molecular Systems Biology, Eidgenössische Technische Hochschule Zürich (ETH Zürich), Zürich, Switzerland

7 Soft Materials Laboratory, Institute of Materials, École Polytechnique Fédérale de Lausanne (EPFL), Lausanne, Switzerland

8 These authors contributed equally. Johannes Bues and Marjan Biočanin

* To whom correspondence should be addressed. Bart Deplancke: bart.deplancke@epfl.ch 


\section{Abstract}

2 Single-cell RNA-sequencing (scRNA-seq) has transformed our ability to resolve cellular

3 properties across systems. However, current scRNA-seq platforms are one-size-fits-all

4 approaches that are tailored toward large cell inputs (> 1,000 cells), rendering them inefficient

5 and costly when processing small, individual tissue samples. This important drawback tends to

6 be resolved by loading bulk samples, but this yields confounded mosaic cell population read-outs.

7 To overcome these technological limitations, we developed a deterministic, mRNA-capture bead

8 and cell co-encapsulation dropleting system, DisCo. We demonstrate that DisCo enables precise

9 particle and cell positioning and droplet sorting control through combined machine-vision and

10 multilayer microfluidics. In comparison to other microfluidics systems, the active flow control

11 driving DisCo, enables continuous operation and processing of low-input samples (<100 cells) at

12 high capture efficiency (> 70\%). To underscore the unique capabilities of our approach, we

13 analyzed intestinal organoid development by "DisCo-ing" 31 individual organoids at varying

14 developmental stages. This revealed extensive organoid heterogeneity, identifying distinct

15 subtypes including a regenerative fetal-like $L y 6 a^{+}$stem cell population which persists as

16 symmetrical cysts even under differentiation conditions. Furthermore, we uncovered a so far

17 uncharacterized "gobloid" subtype consisting predominantly of precursor and mature $\left(\mathrm{Muc2}^{+}\right)$

18 goblet cells. These findings demonstrate the unique power of DisCo in providing high-resolution

19 snapshots of cellular heterogeneity among small, individual tissues. 


\section{Introduction}

28 Single-cell RNA sequencing (scRNA-seq) ${ }^{1}$ induced a paradigm shift in biomedical sciences, since

29 it allows the dissection of cellular heterogeneity by high-dimensional data. Recent technological

30 developments, particularly for cell capture and reaction compartmentalization ${ }^{2-6}$, have led to a

31 substantial increase in experimental throughput, enabling massive mapping efforts such as the

32 mouse and human cell-atlas studies ${ }^{5,7,8}$. These developments were accompanied by biochemical

33 advances, for instance for targeted transcript detection or library multiplexing 9,10 , which present a

34 rich toolbox for large-scale scRNA-seq studies. However, since the majority of methods rely on

35 stochastic cell capture, entailing large sample inputs, efficient processing of small samples (<

361,000 cells) remains challenging. The three main reasons for this are: 1) high fixed run costs,

37 which lead to a large expense per cell at low inputs. For instance, a 10X Chromium run on 100

38 cells would cost $\$ 44$ per sequenced cell. 2) Requirements of minimum cell inputs. For example

39 index-sorting FACS or 10X Chromium require minimum cellular inputs ranging between 10,000

40 and 500 cells, respectively ${ }^{11,12}$. 3) Reduced effectiveness at low inputs because of limited cell

41 capture efficiencies or cell size-selective biases ${ }^{13}$ when processing small heterogeneous

42 samples. To illustrate these limitations, we summarized the performance of various scRNA-seq

43 technologies on low input samples in Supplementary Table 1. Consequently, small samples,

44 involving for instance zebrafish embryos ${ }^{14}$, organisms like C. elegans ${ }^{15}$, or intestinal organoids ${ }^{16-}$

$45^{18}$, are still pooled to obtain cell numbers that are compatible with stochastic microfluidic and well-

46 based technologies. Thus, it is rather paradoxical that limitations overcome by single cell methods

47 are nevertheless reintroduced at the sample level: artificial averages across samples, resulting in

48 an inability to resolve cell type distributions of individual systems or tissues. This particularly

49 hampers research on emergent and self-organizing multicellular systems, such as organoids, that

50 are heterogeneous and small at critical development stages. 
52 In this study, we develop a novel deterministic, mRNA-capture bead and cell co-encapsulation

53 dropleting system (DisCo) for low input scRNA-seq. In contrast to established methods that rely

54 on passive cell capture strategies, we utilize machine-vision to actively detect cells and coordinate

55 their capture in droplets. This active flow control approach allows for continuous operation,

56 enabling free per run scaling and serial processing of samples. We demonstrate that DisCo can

57 efficiently process samples of 100 cells and below, making this platform well suited to handle

58 small, individual tissues. Here, we exploit DisCo's unique capabilities to explore the

59 heterogeneous early development of single intestinal organoids at the single cell level. Grown

60 from single stem cells, organoids of vastly different morphologies and cell type compositions form

61 under seemingly identical in vitro conditions ${ }^{16}$. These unpredictable developmental patterns

62 represent one of the major limitations of this model system, preventing their widespread

63 implementation e.g. in drug screens ${ }^{19}$. Thus, efforts to advance our understanding of the extent

64 of organoid heterogeneity, how it arises, and how it can be controlled, for instance with synthetic

65 growth matrices ${ }^{20,21}$, are of essence. In depth mapping of individual organoid heterogeneity by

66 scRNA-seq has so far been prevented by the minute cell numbers contained in a single intestinal

67 organoid at critical developmental stages, such as post symmetry breaking at the 16-32 cell

68 stage $^{16}$. In total, we "DisCo'd" 31 single organoids at four developmental time points post

69 symmetry breaking, and identified striking differences in cell type composition between individual

70 organoids. Among these subtypes, we detected "spheroids" that are composed of regenerative

71 fetal-like stem cells marked by Stem Cell Antigen-1 (Sca1/Ly6a $)^{22-25}$ and that persist under

72 differentiation conditions. In addition, we uncovered a rare subtype that is predominantly

73 comprised of precursor- and mature goblet cells, which we term "gobloids". 


\section{Results}

79 To develop our Deterministic Co-encapsulation (DisCo) system, we engineered a three inlet

80 (cells, beads, oil) multilayer dropleting device with two outlet ports (sample, waste) (Schematic

81 Figure 1A, full design Supplementary Figure 1A). On this device, each inlet and outlet was 82 augmented with a Quake-style microvalve ${ }^{26}$, to facilitate flow control during operation. In addition, 83 one common valve spanning both the cell and bead channel, termed the dropleting valve, was

84 integrated to allow for on-demand droplet generation. To operate the device, we developed a 85 three-stage process (Figure 1B): 1. Stop two particles at the encapsulation site, 2. Eject particles 86 into one droplet, 3. Selectively extract the droplet in a sample channel (Microscopy images of the 87 process are depicted in Figure 1C). To enable precise coordination of particles in microchannels, we developed a machine-vision-based approach utilizing subsequent image subtraction for blob detection (Supplementary Figure 1B), and on-chip valves for flow-control. Deterministic displacement patterns were induced by opening and closing the cell and bead valves (depicted in Supplementary Figure 1C), which moved particles according to discrete jumps into the target region of interest $(\mathrm{ROI})$ with $95.9 \%$ of particles placed in an approximately $\sim 200 \mu \mathrm{m}$ wide region (Supplementary Figure 1D). Upon placement, the stopped particles were ejected by

94 pressurizing the dropleting valve, displacing an equal volume of liquid from both channels. The 95 ejected liquid phase was then sheared into a droplet by activating the oil stream. We found that 96 precise pressurization of the dropleting valve allowed for accurate control of droplet volume 97 (Supplementary Figure 1E, Supplementary Video 1). Post droplet formation, the outlet valves 98 were actuated to separate the formed droplet from the excess waste liquids (Figure 1D). With all 99 components operating in tight orchestration, we were able to generate monodisperse emulsions 100 with high co-encapsulation purity (Figure 1E, Supplementary Video 2).

102 As a first benchmarking experiment, we set out to determine the encapsulation performance of 103 DisCo for scRNA-seq-related applications, involving co-encapsulation of single cells with 
104 microspheres. Specifically, we aimed to reconfigure the Drop-seq ${ }^{2}$ approach as it only requires 105 coordination of two channels, as compared to three channels for inDrop ${ }^{3}$. Since co-encapsulation 106 purity and cell capture efficiency are critical system parameters for droplet scRNA-seq systems, 107 we quantified the system's processing speed and encapsulation performance in a free-run 108 configuration, i.e. without cell number limitations at varying cell densities. We found that on 109 average, $91.4 \%$ of all droplets contain a cell and a bead, and $1.7 \%$ contain an independent cell 110 doublet (Figure 1F). Overall, the system provided high cell capture efficiencies of $90 \%$ at around 111200 cells per hour for a 2 cells/ $\mu \mathrm{L}$ cell concentration (Figure 1G). At higher cell concentrations of 11220 cells $/ \mu \mathrm{L}$, the processing speed could be increased to 350 cells per hour, yet with decreased 113 capture efficiencies of approximately $75 \%$.

115 Next, we benchmarked the performance of DisCo for scRNA-seq. With drastically reduced bead 116 amounts contained in the generated sample emulsion, we utilized a previously developed chip117 based cDNA generation protocol ${ }^{27}$. Initially, as a library quality measure, we performed a species118 mixing experiment of human HEK 293T and murine brown pre-adipocyte IBA cells. We observed 119 clear species separation (Figure 1H), consistent with the limited number of previously detected 120 doublets (Figure 1F), and increased read-utilization rate compared to conventional Drop-seq 121 experiments (Supplementary Figure 1F). As previously reported ${ }^{28}$, we found that our data 122 displayed a skewed barcode sequence editing distance distribution compared to a true random 123 distribution (Supplementary Figure 1G). Since the uniquely low number of beads in DisCo 124 samples $(<500)$ renders the random occurrence of barcode sequences with an editing distance $125<3$ rare, we developed a graph-based approach to identify and merge closely related barcodes 126 (described in Material and Methods). We found that this approach did not compromise the single 127 cell purity (Supplementary Figure $\mathbf{1 H}$ ) and improved the detectable number of transcripts per 128 cell as compared to published Drop-seq datasets on HEK 293T cells ${ }^{2,27}$ (Figure 1I). 
Since DisCo actively controls fluid flow on the microfluidic device, we observed that the system

131 requires negligible run-in time, and is capable of efficiently processing cells from the first cell on.

132 Given this observation, and the high-capture efficiency of DisCo in free-run mode, we 133 hypothesized that the system should provide reliable performance on small samples of 100 cells 134 and below. To determine the overall cell capture efficiency of DisCo, we precisely quantified the 135 number of input cells using impedance measurements. Specifically, we utilized custom pipette 136 tips augmented with a DISPENCELL gold-plated electrode, which allowed accurate counting of 137 the number of input cells as validated by microscopy (Supplementary Figure 1I). Utilizing the 138 DISPENCELL approach, we processed cell numbers between 50 - 200 cells, of which on average $13986.4 \%$ (SD $\pm 8.1 \%$ ) were visible on the chip. Of all input cells, $79.1 \%(S D \pm 7.4 \%)$ were 140 successfully co-encapsulated, which corresponds to a co-encapsulation efficiency of $91.6 \%$ (SD $141 \pm 1.6 \%$ ) of all visible cells, while $74.9 \%$ (SD $\pm 10.7 \%$ ) of input cells were found as barcodes over 142500 UMls per cell (Figure 1J).

144 As a real-world application, we used DisCo to explore the developmental heterogeneity of 145 intestinal organoids ${ }^{29}$. These polarized epithelial tissues are generated by intestinal stem cells in $1463 \mathrm{D}$ matrices through a stochastic self-organization process, and mimic key geometric, 147 architectural and cellular hallmarks of the adult intestinal mucosa (e.g. a striking crypt-villus-like 148 axis) ${ }^{29}$. When grown from single stem cells, organoids of very different morphologies form under 149 seemingly identical in vitro conditions (Figure 2A, overview image in Supplementary Figure 2A). 150 Pooled tissue scRNA-seq data has shed light on the in vivo-like cell type composition of these 151 organoids ${ }^{16-18,30}$, but cannot resolve inter-organoid heterogeneity. Critical for organoid 152 development is an early symmetry breaking event at Day 2 (16-32 cell stage) that is triggered by 153 cell-to-cell variability and results in the generation of the first Paneth cell responsible for crypt 154 formation ${ }^{16}$. Here, we were particularly interested in examining the emergence of heterogeneity 155 between individual organoids subsequent to the symmetry breaking timepoints. To do so, we 
isolated single LGR5+ cells by FACS, and maintained them in a stem cell state using CHIR99021 and valproic acid $(\mathrm{CV})^{31}$. On Day 3 of culture, $\mathrm{CV}$ was removed to induce differentiation. In total, we sampled 31 single intestinal organoids across four timepoints (Day 3 - 6) (Figure 2A). These organoids were selected based on differences in morphology, and may thus not constitute an unbiased sample of the population. Since Day 3 represents both differentiation Day 0 and the first sampling time point, we re-annotated the data accordingly (S0 - S3 replacing Day 3 - Day 6). During the co-encapsulation run, the number of encapsulated cells was noted and correlated to the number of barcodes retrieved, which was in approximate accordance (Supplementary

164 Figure 2B). The even distribution of the number of reads mapping to ribosomal protein transcripts 165 and the observed low expression of heat shock protein-coding genes indicates that most cells were not affected by dissociation and on-chip processing (Supplementary Figure 2C).

To retrieve a first overview of overall cellular heterogeneity, we jointly visualized all 945 cells passing the quality thresholds through Uniform Manifold Approximation and Projection (UMAP). We found that our data was consistent with previously published pooled organoid scRNA-seq read-outs ${ }^{17,30}$ since it revealed expected cell types including Fabp1-expressing enterocytes, Muc2-expressing goblet cells, Reg3b-positive Paneth cells, and Olfm4-expressing stem cells

173 (Figure 2B and 2C). In addition, a rare subset of cells, likely too few to form clusters, showed 174 ChgA and ChgB expression, indicating the expected presence of enteroendocrine cells 175 (Supplementary Figure 2D). Noteworthy, we found that batch effects are correctable since no 176 batch-based clustering was observed after correction (Supplementary Figure 2E). We also did 177 not detect any clustering driven by cell quality, e.g. detected transcripts or mitochondrial 178 transcripts (Supplementary Figure 2C). These findings support the cell type-resolving power of

179 our DisCo platform (Figure 2C, extensive heatmap in Supplementary Figure 2F, and list in

180 Supplementary Table 2). In addition to the expected cell types, we observed a distinct cluster 181 marked by high expression of Stem cell antigen 1 (Sca1 or Ly6a). In depth analysis of marker 
genes showed high expression of Anxa1 and Clu in the same cluster (Supplementary Figure 2D), and increased YAP-1 target gene expression (Supplementary Figure 2G), suggesting that these cells are most likely regenerative fetal-like stem cells ${ }^{24,25,32}$. Since the two remaining clusters did not show a striking marker gene signature, we resolved their identity by imposing temporal information on the data. This revealed that these clusters likely represent stem- and previously termed potentially intermediate cells $(\mathrm{PIC})^{33}$, given their occurrence at early developmental time points (Figure 2D). As expected, mature cell types were mostly present at later time points. To

189 further leverage the temporal component in the DisCo data, we used slingshot trajectory 190 analysis $^{34}$ to infer lineage relationships between cell types and to identify genes that may be of 191 particular significance for waypoints along differentiation (Figure 2E). Beyond the previously 192 utilized marker genes for cell type annotation, for example Reg3b and Reg3g for Paneth cells, additional established markers ${ }^{35}$ were identified, such as Agr2 and Spink4, and Fcgbp for goblet cells (Figure 2F). Overall, this suggests that the meta-data produced with our DisCo platform aligns with and expands prior knowledge.

Intriguingly, we observed maintained presence of the Ly6a+ stem-cell population at S0, S1, and S3. Since cells with similar expression signatures were previously described under alternate culture conditions as belonging to a distinct organoid subtype termed spheroids ${ }^{23}$, we next aimed to verify the presence of such spheroids among our sampled organoids and study their temporal behavior. To do so, we stratified our cells according to the individual organoids from which they were derived by mapping this information onto the reference scaffold (Figure 3A). Globally, this analysis revealed that the maturation seems to follow the expected pattern with early organoids

205 like goblet cells and enterocytes. However, within single organoids, we found strong 206 heterogeneity, revealing that $L y 6 a^{+}$cells were indeed present in a distinct subset of organoids, 207 predominantly comprised of these cells (S1a, S3e). Furthermore, images obtained prior to 
dissociation showed that $\mathrm{Ly}_{6} \mathrm{a}^{+}$cell-containing organoids (S3e) exhibited a larger, cystic like structure (Supplementary Figure 3A). To confirm the presence of $L y 6 a^{+}$organoids in our

210 cultures, we utilized RNAscope (Figure 3B, controls Supplementary Figure 3B) to localize Ly6a,

211 Muc2, and Fabp1 expression in organoid sections. These analyses revealed canonical budding

212 organoids, containing few Muc2 $2^{+}$goblet cells and Fabp $1^{+}$enterocytes, and Ly6a-expressing cells

213 in spherical organoids that did not contain differentiated cell types such as enterocytes or goblet

214 cells.

216 The presence of $L y 6 a^{+}$cells during the first day of sampling suggested that these cells constitute

217 a second, Lgr5-independent stem cell population in the organoid culture. Using flow cytometry,

218 we found that the majority of cells are either LGR5 ${ }^{+}$LY6A- $^{-}(24.5 \%)$ or LGR5- LY6A $^{+}(3.3 \%)$ with

219 only a minority (0.4\%) being double positive (Figure 3C). This finding, in combination with our

220 trajectory analysis (Figure $2 \mathrm{E}$ and $2 \mathrm{~F}$ ), suggested that $L y 6 \mathrm{a}^{+}$cells are capable of differentiating

221 into organoids. To test this, we sorted and differentiated LGR5- LY6A+ cells, revealing that both

$222 \mathrm{LGR5}^{+} \mathrm{LY} \mathrm{A}^{-}$and LGR5- $\mathrm{LY}^{-} \mathrm{A}^{+}$cells give rise to organoids of similar morphological heterogeneity

223 (Figure 3D). These results indicate that LGR5 $\mathrm{LY}^{-} \mathrm{A}^{+}$cells have full stem cell potential,

224 comparable to that of previously described fetal-like stem cells ${ }^{23}$. Furthermore, the fact that LGR5-

$225 \mathrm{LY}_{6} \mathrm{~A}^{+}$cells did not display a propensity towards spheroid formation suggests that environmental

226 conditions, e.g. matrix stiffness, rather than the initial cell state dictate the formation of spheroids.

228 Beside the $L y 6 \mathrm{a}^{+}$cell-enriched organoids, our data suggested the presence of additional organoid 229 subtypes in the per organoid mappings (Figure 3A). The two most striking additional subtypes 230 were three organoids that contained mostly enterocytes (S2c, S3a, S3d), and two that consisted 231 predominantly of immature and mature goblet cells ( $\mathrm{S} 1 \mathrm{~b}$ and especially $\mathrm{S} 2 \mathrm{f})$. The identity of the 232 observed subtypes was further substantiated when visualizing the cell type abundance per 233 organoid (Figure 3E), and marker gene expression in individual organoids (Supplementary 
234 Figure 3C). Similar to the spheroids, both subtypes showed aberrant morphologies, tending to

235 be small and round, as compared to canonical organoids bearing a crypt-villus axis (e.g. S3c,

236 Supplementary Figure 3A). To detect more subtle molecular differences, we used psupertime ${ }^{36}$

237 to identify genes that are dynamically expressed during the development of individual organoids.

238 This analysis revealed additional genes that are expressed in subsets of organoids, such as

239 Gastric inhibitory polypeptide (Gip), Zymogen granule protein 16 (Zg16), Vanin 1 (Vnn1), and

240 Defensin alpha 24 (Defa24) (Supplementary Figure 3D).

242 While organoids dominated by enterocytes were previously described as enterocysts ${ }^{16}$, organoids

243 displaying goblet cell hyperplasia, here termed "gobloids", were so far to our knowledge unknown.

244 To validate the existence of the uncovered organoid subtypes, we utilized RNAscope to localize

245 the expression of enterocyte (Fabp1) and goblet cell (Muc2) markers (Figure 3F, controls in

246 Supplementary Figure 3B). In addition, and in agreement with our data and prior research, we

247 detected organoids that exclusively contained Fabp $1^{+}$cells, most likely representing enterocysts.

248 Most importantly, we were able to identify organoids that contained a high number of $\mathrm{Muc2}^{+}$goblet

249 cells, confirming the existence of "gobloids".

\section{Discussion}

252 A key feature of our new DisCo approach is the ability to deterministically control the cell capture

253 process. Despite lowering the throughput compared to stochastic droplet systems ${ }^{2,3}$, our 254 approach provides the advantage of being able to process low cell input samples at high efficiency 255 and at a strongly decreased per cell cost (Supplementary Table 1). Thus, we believe that the 256 DisCo approach is filling an important gap in the scRNA-seq toolbox. Moreover, full control over

257 the encapsulation process allows for continuous operation of our platform, which is offsetting to 258 some extent the decreased throughput. Another critical feature of DisCo is the use of machine259 vision to obtain full control of the entire co-encapsulation process including particle detection, 
260 particle positioning, particle droplet injection, and droplet volume. This enables the correct 261 assembly of most droplets, virtually eradicating confounding factors that arise due to failed co262 encapsulations ${ }^{37,38}$. In concept, DisCo is thus fundamentally different to passive particle pairing 263 approaches such as traps ${ }^{39-41}$ and, compared to these technologies, offers the advantage of 264 requiring vastly simpler and reusable chips without suffering from cell/particle size and shape 265 selection biases ${ }^{13,42}$. This renders the DisCo approach universally applicable to any particle co266 encapsulation application ${ }^{43,44}$, i.e. cell-cell encapsulations, with the only limiting factor being 267 particle visibility. Providing further development, we envision that machine learning-based 268 deterministic cell handling will ultimately enable targeted cell selection, e.g. by fluorescence or 269 morphology, transforming DisCo into an end-to-end cell processor for samples with low-to270 medium input samples.

272 To demonstrate DisCo's capacity to process small tissues/systems that were so far difficult to 273 access experimentally, we have analyzed the cell heterogeneity of chemosensory organs from 274 Drosophila larvae ${ }^{45}$ and, as shown here, single intestinal organoids. It is thereby worth noting that, 275 based on our handling of distinct tissues, we found that not DisCo itself, but rather cell dissociation 276 has become the efficiency-limiting factor, a well-recognized challenge in the field ${ }^{46,47}$. Indeed, 277 substantial cell loss was a regular occurrence, even with optimized dissociation and processing 278 strategies (see Methods).

280 scRNA-seq of individual organoids led us to uncover organoid subtypes of aberrant cell type 281 distribution that were previously not resolved with pooled organoid scRNA-seq ${ }^{16,17,30}$. One subtype 282 contained predominantly cells that were strikingly similar to previously described fetal-like stem 283 cells or revival stem cells that occur during intestinal regeneration ${ }^{24,25,32}$. This subtype, previously 284 described under alternate culture conditions as spheroid-type organoids ${ }^{18,22,23}$, was identified here 285 under standard organoid differentiation conditions, indicating that these organoids are capable of 
maintaining their unique state. We isolated LY6A-expressing cells and found that they readily give rise to canonical organoids, indicating that these cells are capable of providing a pool of multipotent stem-cells. Of particular interest was one organoid subtype that we termed "gobloid" given that it predominantly comprises immature and mature goblet cells. Since low Notch signaling is pivotal for the commitment of crypt base columnar (CBC) cells towards secreting progenitors, lack of Notch ligand-providing Paneth cells ${ }^{48}$, may drive gobloid development ${ }^{49}$. However, failure to produce Paneth cells has previously been suggested as a mechanism underlying enterocyst development ${ }^{16}$, which in principle requires high Notch signaling. Hence, we

294 believe that our findings establish an important foundation to support further research on the 295 emergence of gobloids and enterocysts from the still elusive PIC cells, providing an exciting opportunity to delineate lineage commitment factors of CBC cell differentiation.

In sum, we demonstrate that our DisCo analysis of individual organoids is a powerful approach to explore tissue heterogeneity and to yield new insights into how this heterogeneity arises. In comparison to established approaches such as automated microscopy ${ }^{16,18}$, DisCo is magnitudes lower in experimental scale. Nevertheless, scRNA-seq data acquired from 31 organoids enabled us to recapitulate previous findings, benchmarking DisCo, and most importantly, to uncover novel subtypes, leveraging the key advantage of scRNA-seq, i.e. independence from a priori knowledge. Next to catalyzing research on other tissues or systems of interest, we believe that the technology and findings of this study will contribute to future research on intestinal organoid development and thus aid the engineering of more robust organoid systems. Furthermore, we

307 expect this approach to be applicable to rare, small clinical samples to gain detailed insights into 308 disease-related cellular heterogeneity and dynamics. 


\section{Acknowledgements}

313 We thank Wanze Chen and Petra C. Schwallie for constructive discussions. We thank Virginie

314 Braman for help in establishing intestinal organoid culture in our group, and Giovanni Sorrentino

315 from Kristina Schoonjans' lab for valuable advice and support during organoid culture

316 establishment. We also thank Luc Aeberli and Georges Muller from SEED Biosciences for cell

317 sorting support. We thank the EPFL CMi, GECF, BIOP, FCCF, Histology core facility, SCITAS,

318 and UNIL VITAL-IT for device fabrication, sequencing, imaging, sorting, histology, and

319 computational support respectively. We particularly thank Jessica Sordet-Dessimoz for her

320 support with the RNA-scope assay. This research was supported by an Animalfree Research 3R

321 Grant, the Swiss National Science Foundation Grant (IZLIZ3_156815) and a Precision Health \&

322 related Technologies (PHRT-502) grant to B. D., the Swiss National Science Foundation SPARK

323 initiative (CRSK-3_190627) and the EuroTech PostDoc programme co-funded by the European

324 Commission under its framework programme Horizon 2020 (754462) to J. P., as well as by the 325 EPFL SV Interdisciplinary PhD Funding Program to B. D. and E. A.. Y.S. is an ISAC Marylou 326 Ingram scholar.

\section{Contributions}

$329 \mathrm{BD}, \mathrm{JB}$, and $\mathrm{MB}$ designed the study. $\mathrm{BD}, \mathrm{JB}, \mathrm{MB}$, and JP wrote the manuscript. JB and RD 330 designed and fabricated microfluidic chip. JB developed the machine-vision integration for DisCo.

$331 \mathrm{JB}$ and MB benchmarked the system and performed all single-cell RNA-seq experiments. JP, JB,

$332 \mathrm{MB}, \mathrm{WS}, \mathrm{VG}$, and $\mathrm{RG}$ performed data analysis related to single organoid scRNA-seq 333 experiments. JB, SR and MB performed all organoid and cell culture assays. JB, AC, and JR, 334 performed all imaging assays. EA provided critical comments regarding microfluidic chip design 335 and fabrication. MC provided critical comments on intestinal organoid scRNA-seq data analysis.

336 ML provided critical comments regarding intestinal organoid scRNA-seq data and design of critical 337 confirmation experiments. All authors read, discussed, and approved the final manuscript. 


\section{References}

1. Tang, F. et al. mRNA-Seq whole-transcriptome analysis of a single cell. Nat. Methods 6, 377-382 (2009).

2. Macosko, E. Z. et al. Highly parallel genome-wide expression profiling of individual cells using nanoliter droplets. Cell 161, 1202-1214 (2015).

3. Klein, A. M. et al. Droplet barcoding for single-cell transcriptomics applied to embryonic stem cells. Cell 161, 1187-1201 (2015).

4. Gierahn, T. M. et al. RNA sequencing of single cells at high throughput. Nat. Methods 14, 395-398 (2017).

5. Han, X. et al. Mapping the Mouse Cell Atlas by Microwell-Seq. Cell 172, 1091-1107 (2018).

6. Rosenberg, A. B. et al. Single-cell profiling of the developing mouse brain and spinal cord with split-pool barcoding. Science 360, 176-182 (2018).

7. The Tabula Muris Consortium. Single-cell transcriptomics of 20 mouse organs creates a Tabula Muris. Nature 562, 367-372 (2018).

8. Han, X. et al. Construction of a human cell landscape at single-cell level. Nature (Springer US, 2020). doi:10.1038/s41586-020-2157-4

9. Saikia, M. et al. Simultaneous multiplexed amplicon sequencing and transcriptome profiling in single cells. Nat. Methods 16, 59-62 (2019).

10. Gehring, J., Hwee Park, J., Chen, S., Thomson, M. \& Pachter, L. Highly multiplexed single-cell RNA-seq by DNA oligonucleotide tagging of cellular proteins. Nat. Biotechnol. 38, 35-38 (2020).

11. Hwang, B., Lee, J. H. \& Bang, D. Single-cell RNA sequencing technologies and bioinformatics pipelines. Exp. Mol. Med. 50, 1-14 (2018).

12. 10X Genomics. Chromium Single Cell 3' Reagent Kits v3. 1000153, 1-55 (2018).

13. DeLaughter, D. M. The Use of the Fluidigm C1 for RNA Expression Analyses of Single Cells. Curr. Protoc. Mol. Biol. 122, 1-17 (2018).

14. Wagner, D. E. et al. Single-cell mapping of gene expression landscapes and lineage in the zebrafish embryo. Science 360, 981-987 (2018).

15. Packer, J. S. et al. A lineage-resolved molecular atlas of C. Elegans embryogenesis at single-cell resolution. Science 365, (2019).

16. Serra, D. et al. Self-organization and symmetry breaking in intestinal organoid development. Nature 562, 66-72 (2019).

17. Grün, D. et al. Single-cell messenger RNA sequencing reveals rare intestinal cell types. Nature 525, 251-255 (2015).

18. Lukonin, I. et al. Phenotypic landscape of intestinal organoid regeneration. Nature 586, 275-280 (2020).

19. Rossi, G., Manfrin, A. \& Lutolf, M. P. Progress and potential in organoid research. Nat. Rev. Genet. 19, 671-687 (2018).

20. Gjorevski, N. et al. Designer matrices for intestinal stem cell and organoid culture. Nature 539, 560-564 (2016).

21. Brassard, J. A. \& Lutolf, M. P. Engineering Stem Cell Self-organization to Build Better Organoids. Stem Cell 24, 860-876 (2019).

22. Mustata, R. C. et al. Identification of Lgr5-Independent Spheroid-Generating Progenitors of the Mouse Fetal Intestinal Epithelium. Cell Rep. 5, 421-432 (2013).

23. Yui, S. et al. YAP / TAZ-Dependent Reprogramming of Colonic Epithelium Links ECM Remodeling to Tissue Regeneration. Cell Stem Cell 22, 35-49 (2018).

24. Ayyaz, A. et al. Single-cell transcriptomes of the regenerating intestine reveal a revival stem cell. Nature 569, 121-125 (2019).

25. Roulis, M. et al. Paracrine orchestration of intestinal tumorigenesis by a mesenchymal niche. Nature 580, 524-529 (2020).

26. Unger, M. A., Chou, H. P., Thorsen, T., Scherer, A. \& Quake, S. R. Monolithic microfabricated valves and pumps by multilayer soft lithography. Science 288, 113-116 (2000).

27. Biocanin, M., Bues, J., Dainese, R., Amstad, E. \& Deplancke, B. Simplified Drop-seq workflow with minimized bead loss using a bead capture and processing microfluidic chip. LoC 19, 1610-1620 (2019).

28. Zhang, X. et al. Comparative Analysis of Droplet-Based Ultra-High-Throughput Single-Cell RNASeq Systems. Mol. Cell 1-13 (2018). doi:10.1016/j.molcel.2018.10.020 
29. Sato, T. et al. Single Lgr5 stem cells build crypt - villus structures in vitro without a mesenchymal niche. Nature 459, 262-266 (2009).

30. Haber, A. L. et al. A single-cell survey of the small intestinal epithelium. Nature 551, 333-339 (2017).

31. Yin, X. et al. Niche-independent high-purity cultures of Lgr5 + intestinal stem cells and their progeny. Nat. Methods 11, 106-112 (2014).

32. Gregorieff, A., Liu, Y., Inanlou, M. R., Khomchuk, Y. \& Wrana, J. L. Yap-dependent reprogramming of Lgr5+ stem cells drives intestinal regeneration and cancer. Nature 526, 715718 (2015).

33. Battich, N. et al. Sequencing metabolically labeled transcripts in single cells reveals mRNA turnover strategies. Science 367, 1151-1156 (2020).

34. Street, K. et al. Slingshot : cell lineage and pseudotime inference for single-cell transcriptomics. BMC Genomics 19, 1-16 (2018).

35. Birchenough, G., Johansson, M., Gustafsson, J., Bergstrom, J. \& Hansson, G. C. New developments in goblet cell mucus secretion and function. Mucosal Immunol. 8, 712-719 (2015).

36. Macnair, W. \& Claassen, M. psupertime: supervised pseudotime inference for single cell RNA-seq data with sequential labels. bioRxiv 622001 (2019). doi:10.1101/622001

37. Lareau, C. A., Ma, S., Duarte, F. M. \& Buenrostro, J. D. Inference and effects of barcode multiplets in droplet-based single-cell assays. Nat. Commun. 11, 1-9 (2020).

38. Lun, A. T. L. et al. EmptyDrops: Distinguishing cells from empty droplets in droplet-based singlecell RNA sequencing data. Genome Biol. 20, 1-9 (2019).

39. Chung, M., Núñez, D., Cai, D. \& Kurabayashi, K. Deterministic droplet-based co-encapsulation and pairing of microparticles via active sorting and downstream merging. Lab Chip 17, 3664-3671 (2017).

40. Cheng, Y. H. et al. Hydro-Seq enables contamination-free high-throughput single-cell RNAsequencing for circulating tumor cells. Nat. Commun. 10, 1-11 (2019).

41. Zhang, M. et al. Highly parallel and efficient single cell mRNA sequencing with paired picoliter chambers. Nat. Commun. 11, 1-13 (2020).

42. Pollen, A. A. et al. Low-coverage single-cell mRNA sequencing reveals cellular heterogeneity and activated signaling pathways in developing cerebral cortex. Nat. Biotechnol. 32, 1053-1058 (2014).

43. Dura, B. et al. Profiling lymphocyte interactions at the single-cell level by microfluidic cell pairing. Nat. Commun. 6, 1-13 (2015).

44. Gérard, A. et al. High-throughput single-cell activity-based screening and sequencing of antibodies using droplet microfluidics. Nat. Biotechnol. 38, 715-721 (2020).

45. Maier, G. L. et al. Multimodal and multisensory coding in the Drosophila larval peripheral gustatory center. bioRxiv (2020). doi:10.1101/2020.05.21.109959

46. Haque, A., Engel, J., Teichmann, S. A. \& Lönnberg, T. A practical guide to single-cell RNAsequencing for biomedical research and clinical applications. Genome Med. 9, 1-12 (2017).

47. Denisenko, E. et al. Systematic assessment of tissue dissociation and storage biases in single-cell and single-nucleus RNA-seq workflows. Genome Biol. 21, 1-25 (2020).

48. Sato, T. et al. Paneth cells constitute the niche for Lgr5 stem cells in intestinal crypts. Nature 469, 415-418 (2011).

49. Van Es, J. H. et al. Notch/Y-secretase inhibition turns proliferative cells in intestinal crypts and adenomas into goblet cells. Nature 435, 959-963 (2005). 
A

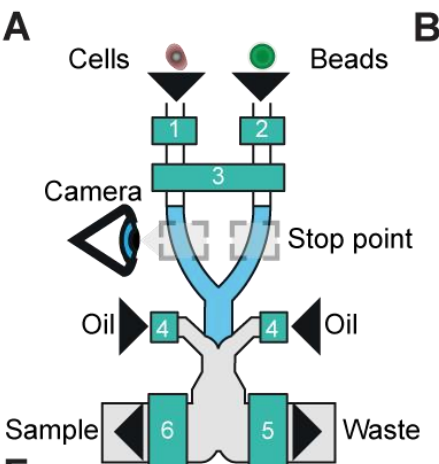

E

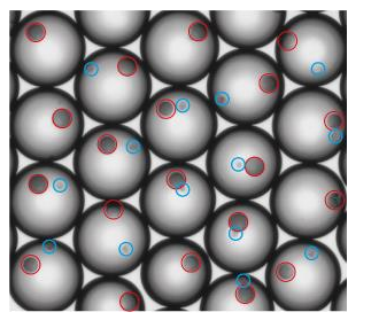

F

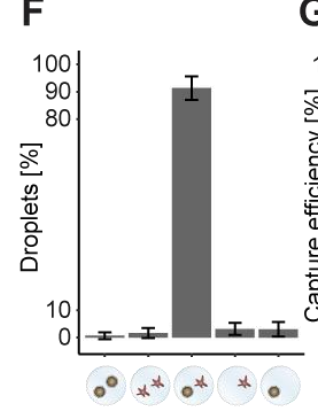

G
B
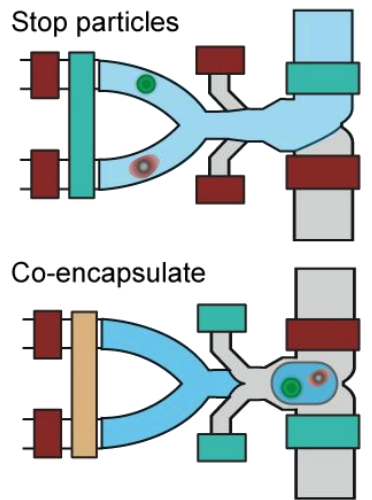

Capture

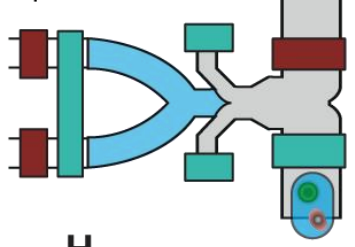

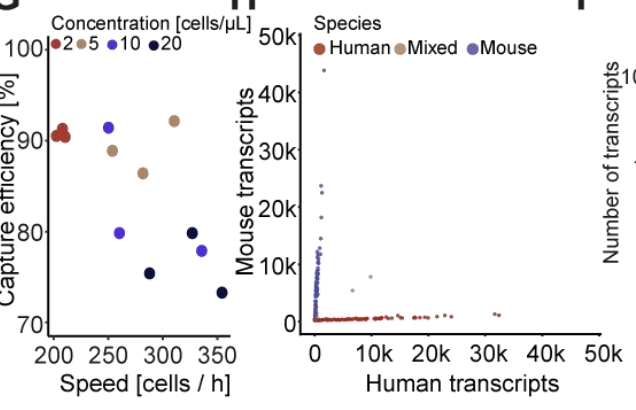

C

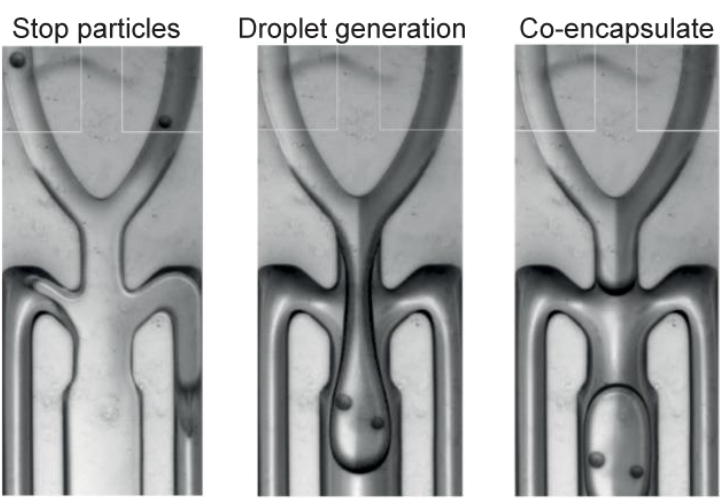

D

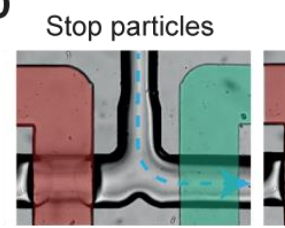

Wash channel
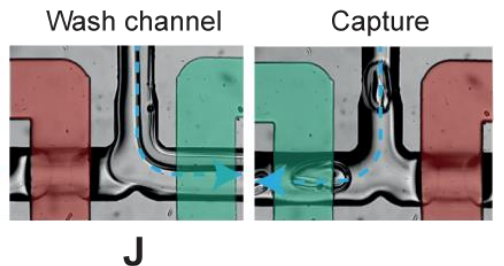

J
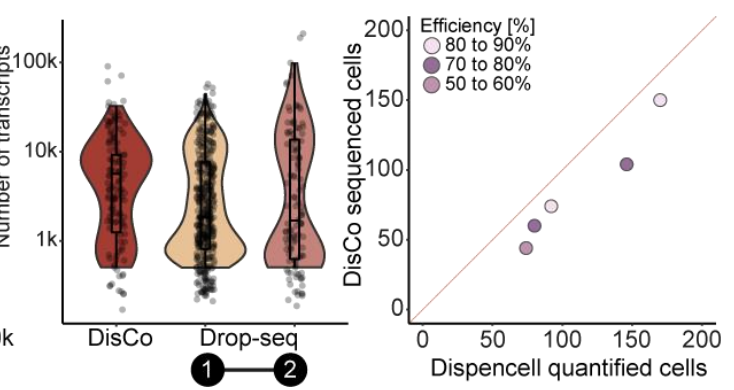

Figure 1. Overview and critical feature assessment of the deterministic co-encapsulation (DisCo) system: (A) Schematics of the DisCo microfluidic device. The device contains three inlet channels for cells, beads, and oil, and two outlets for waste and sample liquids. All inlets and outlets are augmented with Quake-style microvalves (green boxes): 1. cell valve, 2. bead valve, 3. dropleting valve, 4. oil valve, 5 . waste valve, 6 . sample valve. The device is continuously monitored by a high-speed microscopy camera to detect and coordinate placement of particles at the Stop point. (B) Illustration of the particle coencapsulation process on the DisCo device. Initially, two particles (here a bead and a cell) are stopped (Stop particles) in close proximity to the channel junctions by closing the channel valves (red: closed, green: open). Next, by pressurizing the dropleting valve (yellow), both particles are ejected into the junction point, and the droplet is sheared by opening the oil valve (Co-encapsulate). Finally, the produced droplet is captured in the Sample channel (Capture). (C) The co-encapsulation process of two beads and droplet generation as observed on chip. Dyed liquids were used to examine the liquid interface of the carrier liquids. Channel sections with white squares are $100 \mu \mathrm{m}$ wide. (D) The droplet capture process as observed onchip. Valves are highlighted according to their actuation state (red: closed, green: open). While particles are stopped, excess buffers are discarded through the waste channel and the channel is flushed with oil prior to droplet capture. Upon co-encapsulation, the waste valve is closed, the sample valve opened, and the produced droplet captured in the Sample channel. (E) Images of DisCo droplet contents. Cells (blue circle) and beads (red circle) were co-encapsulated, and captured droplets imaged. Mean bead-size is approximately $30 \mu \mathrm{m}$. (F) Droplet occupancy of DisCo-processed cells and beads for cell concentrations ranging from 2 to 20 cells per $\mu$ ( (total encapsulations $n=1203$ ). Error bars represent standard deviation. (G) Cell capture efficiency and cell capture speed for varying cell concentrations (total encapsulations $n=$ 1203). Cells were co-encapsulated with beads at concentrations ranging from $2-20$ cells per $\mu \mathrm{l}$, and coencapsulation events quantified by analyzing recordings of the process. (H) DisCo scRNA-seq species separation experiment. HEK $293 \mathrm{~T}$ and murine pre-adipocyte iBA cells were processed with the DisCo workflow for scRNA-seq, barcodes merged, and species separation visualized as a Barnyard plot. (I) 
471 Comparison of detected UMls per cell of conventional Drop-seq experiments. UMls per cell from HEK 293T 472 data for conventional Drop-seq experiments ([1] - from Biočanin, Bues et al. 201927 and [2] - from Macosko 473 et al. 20152), compared to the barcode-merged HEK 293T DisCo data. Drop-seq datasets were down474 sampled to comparable sequencing depth. Box elements are described in the Materials and Methods 475 section. (J) Total cell processing efficiency of DisCo at low cell inputs. Input cells (HEK 293T) ranging from 47674 to 170 were quantified with the Dispencell system. Subsequently, all cells were processed with DisCo, 477 sequenced, and quality filtered (> 500 UMIs). The red line represents $100 \%$ efficiency, and samples were 478 colored according to recovery efficiency after sequencing. 
A

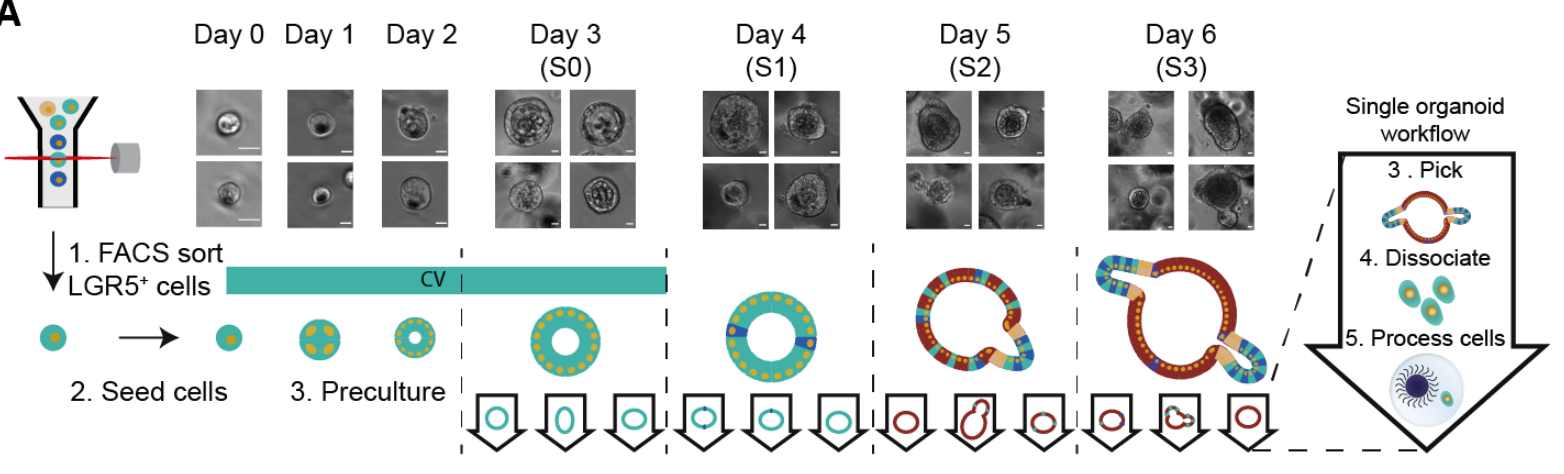

B

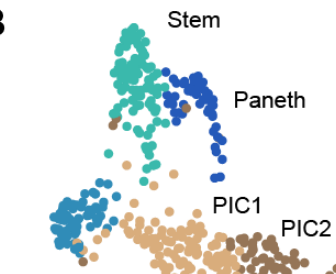

C
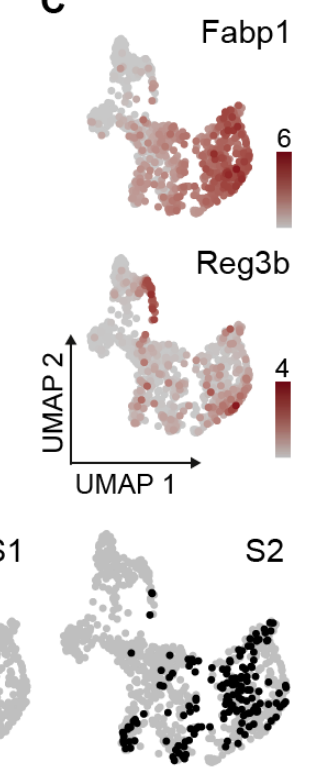

S1

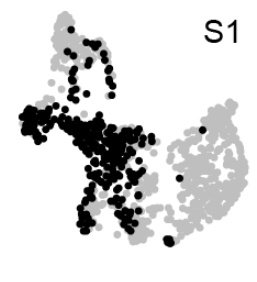

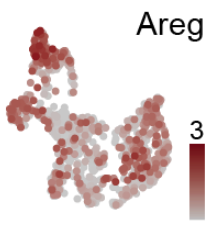
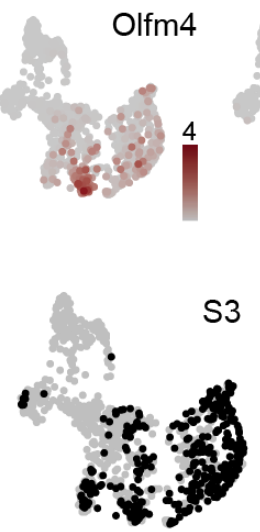
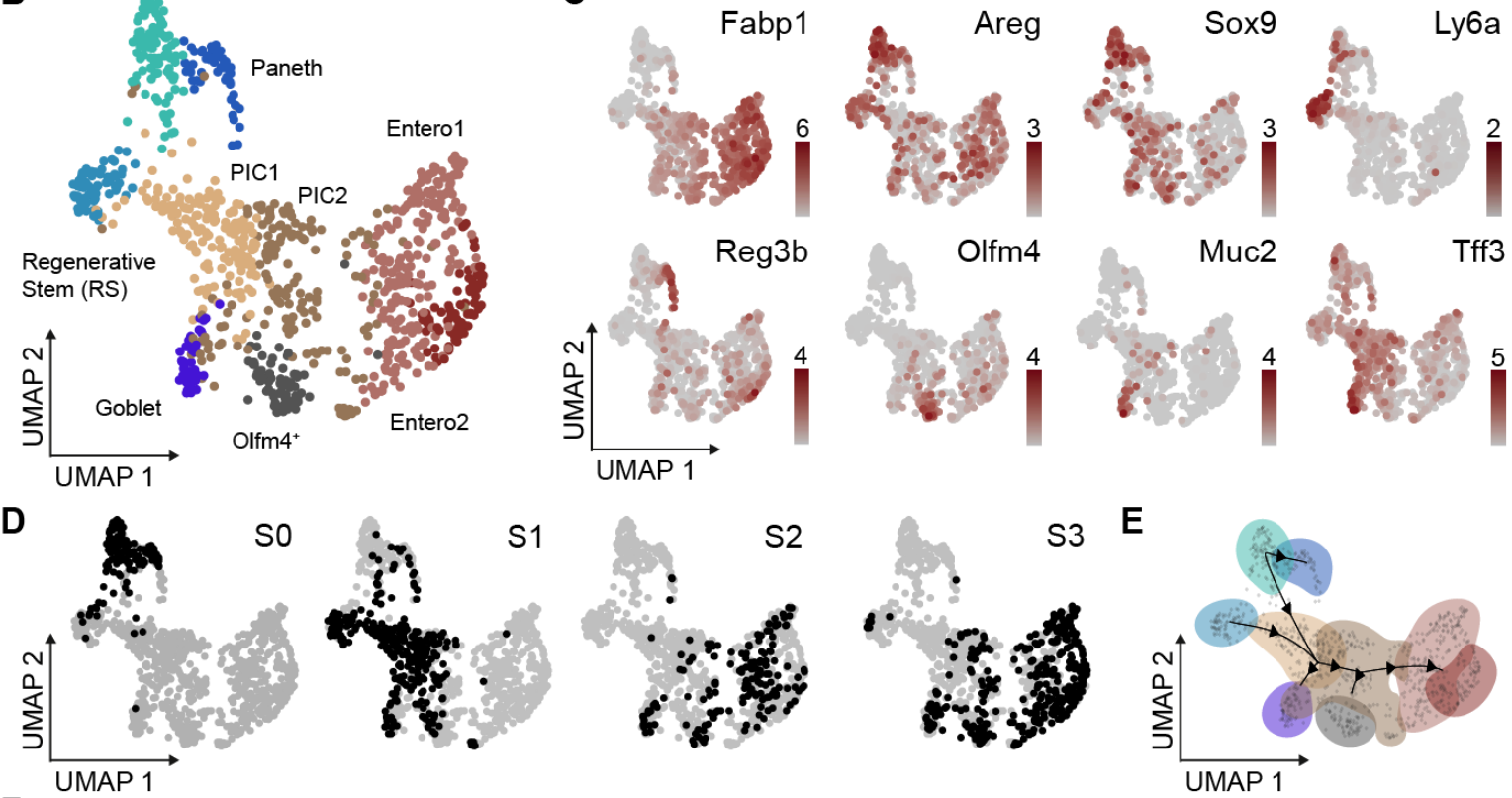

$\mathbf{F}$
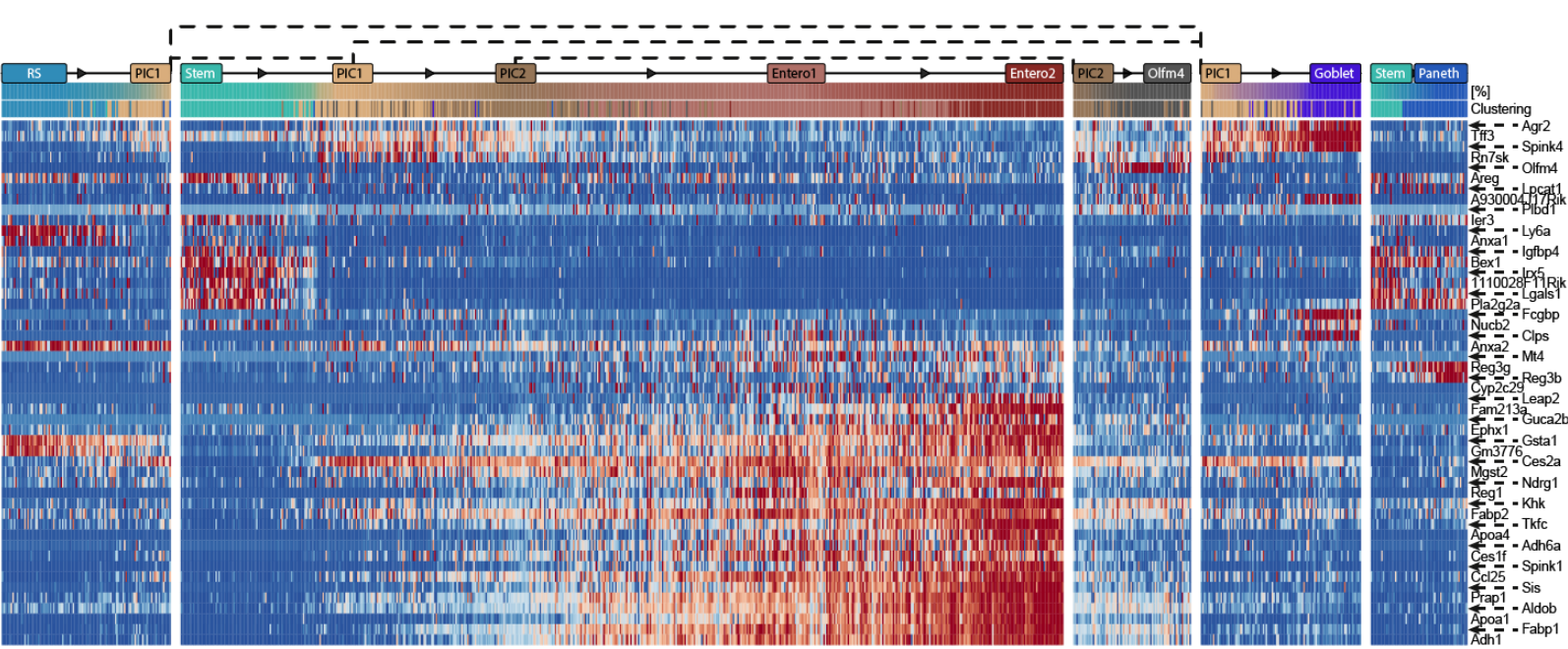

E

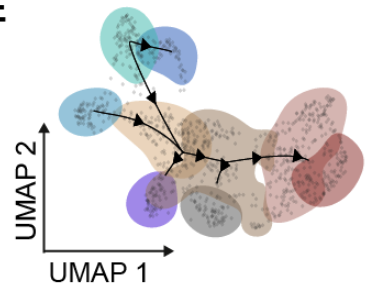

Figure 2. Utilizing DisCo to map intestinal organoid cell heterogeneity along development: (A) Overview of the experimental design for DisCo'ing individual organoids. Single LGR5+ intestinal stem cells were isolated via FACS and precultured for 3 days under stem cell maintenance conditions (ENR CV Day 0 to 3). On Day 3, CV was removed from the culture, and organoids differentiated under ENR conditions for up to 3 days. For each day during development (SO - S3), individual organoids were isolated, dissociated, and processed on the DisCo platform. Representative bright-field imaging examples of 
487 individual organoids for each day are shown on top. Scale bar $50 \mu \mathrm{m}$. (B) UMAP embedding of all 488 sequenced cells. All 945 processed cells from 31 organoids were clustered with k-means clustering, after 489 which clusters were annotated according to marker gene expression. (C) UMAP-based visualization of the 490 expression of specific markers that were used for cluster annotation. (D) Temporal occurrence of cells. 491 Cells are highlighted on the UMAP embedding according to sampling time point (S0 - S3). (E) 492 Developmental trajectory based on the cluster annotation and the sampling time point derived by slingshot ${ }^{34}$. Cells were annotated in accordance with clustering in (B). (F) Heat map of differentially expressed genes along the waypoints of the trajectory. Waypoints are annotated in accordance with cell clustering as in (B). Cluster abbreviations: Stem cells (Stem), Regenerative stem cells (RS), Potential intermediate cells (PIC) ${ }^{33}$, Enterocytes cluster 1/2 (Entero1/2). 
A

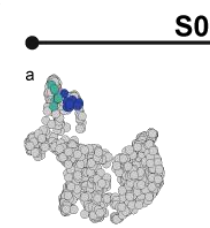

so
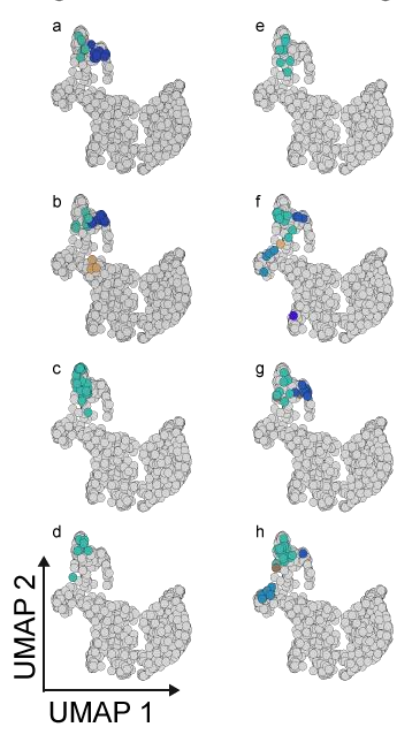

B
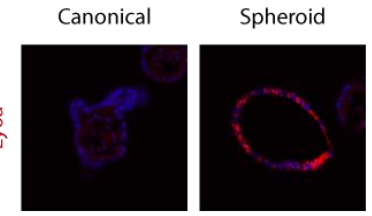

$\frac{\overline{0}}{\frac{0}{10}}$
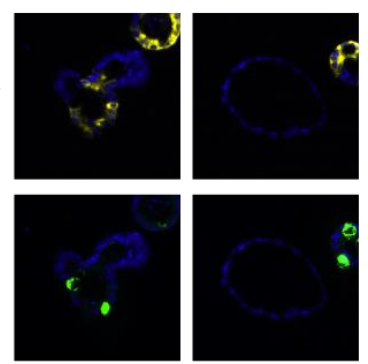

$\mathbf{F}$

Enterocyst
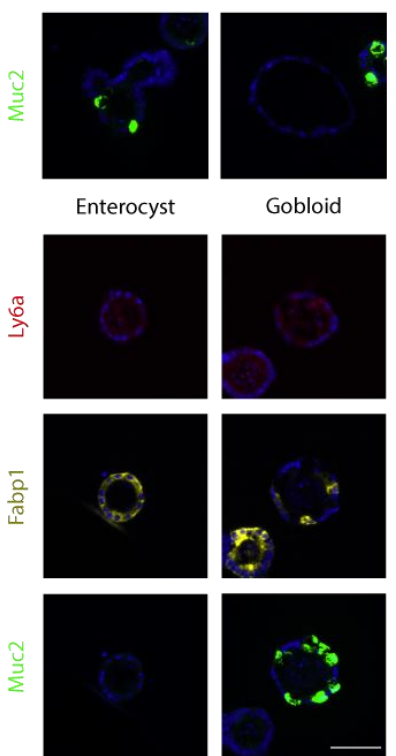
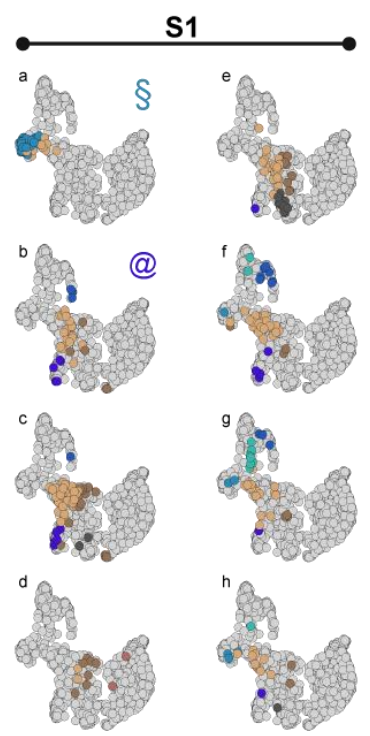

S2
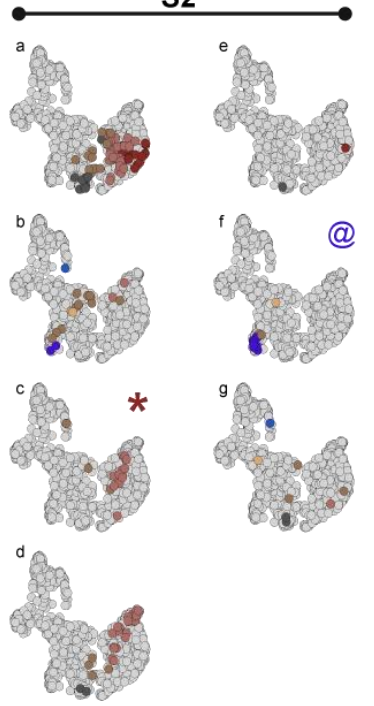
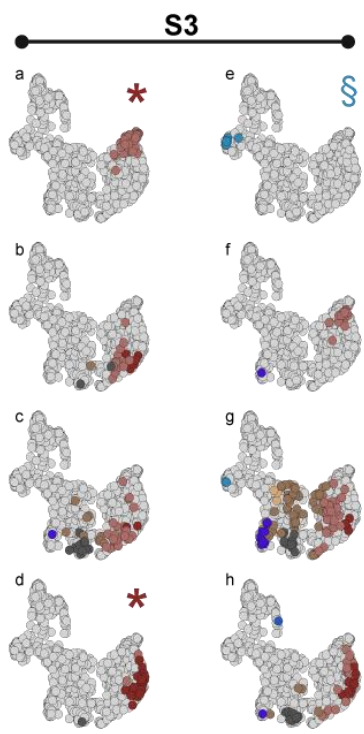

Cluster Stem Regenerative Stem 9 Paneth $P$ PIC 1 PIC $2 \bigcirc$ Goblet $\bigcirc$ Olfm4 $4^{+}$Entero 1 Entero 2

Gobloid
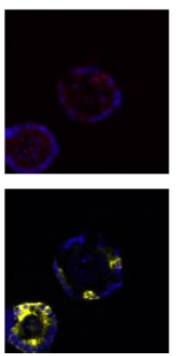

C

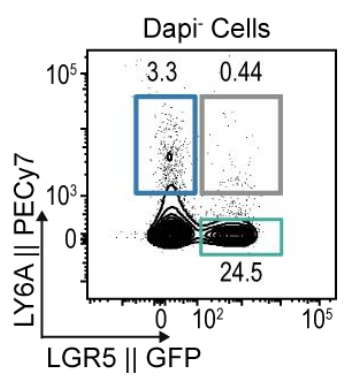

E

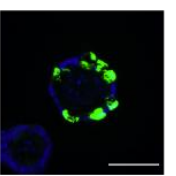

\section{So}

D
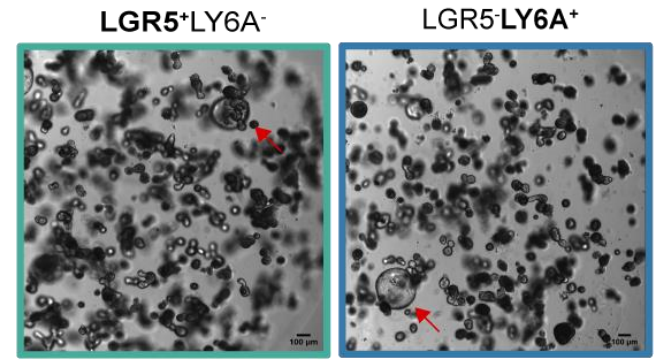

S1

S2

S3

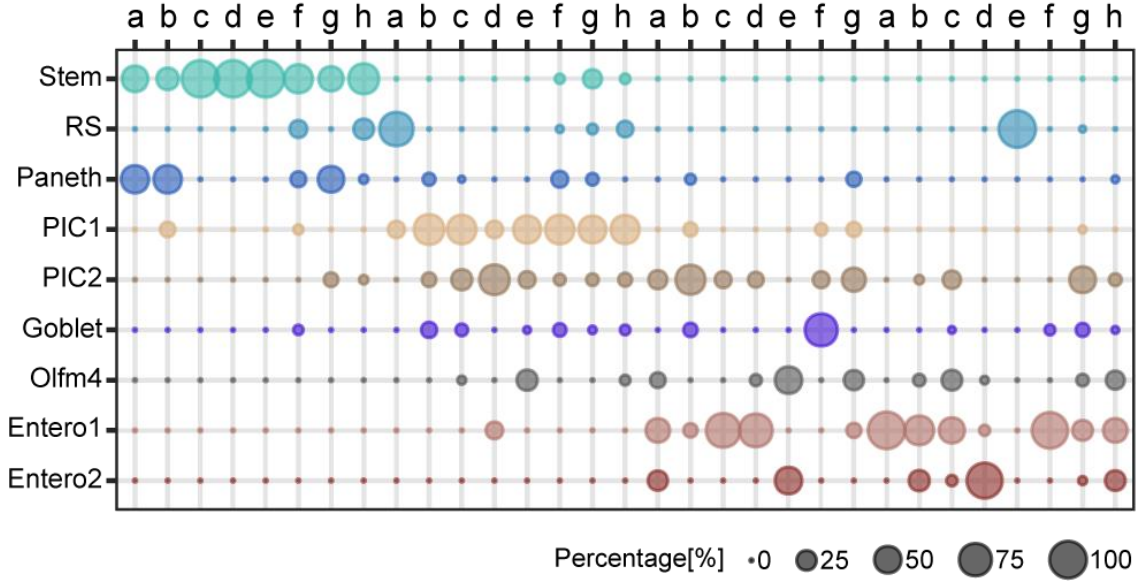

Figure 3. Cell type distribution and marker gene expression across individual intestinal organoids

during development: (A) Projection of cell types onto 31 individual organoids. Cells per single organoid were colored according to their global clustering and highlighted on the UMAP embedding of all sequenced cells. Projections are grouped according to their sampling time. Manually classified organoids were annotated with the following symbols: "*” enterocysts, "§” spheroids, “@” gobloids. (B) in situ RNA detection 
504 of Ly6a, Fabp1, and Muc2 expression. A representative canonical and Ly6a-expressing organoid is 505 displayed. Scale bar (displayed in F) $50 \mu \mathrm{m}$. (C) Surface LY6A and LGR5-GFP expression under ENR CV 506 conditions. The dot plot depicts LGR5-GFP and LY6A expression in organoid-derived single cell 507 suspensions. The numbers indicate frequencies (\%). (D) Culturing outcomes of LGR5+cells and LY6A+ cells. Single LGR5 ${ }^{+} \mathrm{LY}^{-}$and $\mathrm{LGR}^{-} \mathrm{LY}^{-} \mathrm{A}^{+}$cells were isolated by FACS and seeded in Matrigel. Cells were cultured as depicted in Figure 2a and imaged using bright-field microscopy at S3. Red arrows point to spheroid morphologies. Scale bar $100 \mu \mathrm{m}$. (E) Dotplot depicting the distribution of annotated cell types per organoid. Dot size depicts the percentage of cells associated to each cluster per organoid. (F) in situ RNA detection of Fabp1 and Muc2 expression. Selected images resembling the enterocyst and gobloid subtypes. Scale bar $50 \mu \mathrm{m}$. 